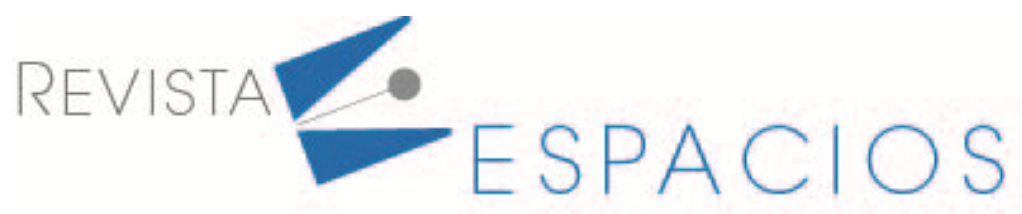

\title{
Transformación digital y enfoque STEAM, una alternativa en tiempos de COVID-19
}

\section{Digital transformation and STEAM approach, an alternative in times of COVID-19.}

\author{
VARGAS, Javier ${ }^{1}$ \\ CUERO, Jairo ${ }^{2}$ \\ RIVEROS, Fernando ${ }^{3}$
}

\section{Resumen}

El artículo presenta los resultados de una investigación en el marco del enfoque STEAM y la transformación digital como alternativas para implementar en los sistemas educativos actuales y después de la pandemia ocasionada por el COVID-19. La metodología utilizada fue análisis de contenido y entrevista a profundidad realizada a expertos académicos. Los resultados obtenidos permitieron concluir que el enfoque STEAM y la transformación digital son pertinentes y apropiadas para el momento actual y futuro de la sociedad del siglo XXI.

Palabras clave: covid -19, steam, transformación digital, blended learning, e-learning, educación en pandemia

\begin{abstract}
This paper presents the results of research in the framework of the STEAM approach and the digital transformation as a curricular alternative to apply in the educational systems during and after the Covid-19 pandemic. The methodology used was content analysis and in-depth interviews with academic experts. The results obtained allowed us to conclude that the STEAM approach and the digital transformation are relevant and appropriate for the current and future moment of the 21stcentury society.
\end{abstract}

key words: covid -19 , steam, digital transformation, blended learning, e-learning, pandemic education

\section{Introducción}

Con el estado de emergencia mundial generado por el COVID -19 la educación mediada por tecnologías aumentó significativamente, al punto de convertir cada espacio con acceso de internet en el hogar como un aula de clase y ocasionando un impacto en los actores del proceso educativo. Como resultado de este impacto, ahora se tiene estudiantes más conectados interactuando con profesores y padres de familia, lo que genera espacios de aprendizaje complejos, pero con un potencial alto (Argudo \& Tenecela, 2019) (Wosik et al., 2020).

\footnotetext{
${ }^{1}$ Doctor En ciencias de la Educación - Investigador del grupo Macrypt y del grupo EYSI. Javier.andres.vargas@unillanos.edu.co

${ }^{2}$ Candidato a Magister en Automatización - Investigador del grupo Macrypt y del grupo EYSI. Jairo_cuero@unilllanos.edu.co

${ }^{3}$ Estudiante de ingeniería electrónica - Universidad de los Llanos
} 
No obstante, estos escenarios complejos de aprendizaje no son nuevos y han venido evolucionando de tal manera que antes de la pandemia existían enfoques educativos como el STEAM, el cual permite integrar los conocimientos de las matemáticas, ciencias, tecnologías, arte, ingeniería y matemáticas para el desarrollo de habilidades científicas y tecnológicas que aportan a la toma de decisiones asertivas que permitan brindar soluciones reales al contexto de cada estudiante, promoviendo el aprendizaje a lo largo de toda la vida, como lo plantea la UNESCO (Habib, González, Collazos, \& Yousef, 2020).

Adicionalmente, aunque se reconoce que la educación STEAM es una propuesta pertinente para afrontar los procesos educativos en tiempos del COVID 19, es necesario analizar el contexto ya que no en todos se presentan las condiciones mínimas para su implementación. Por ejemplo, países como los del continente africano presentan una necesidad tecnológica significativa que obstaculiza los procesos de educación E-learning, Blearning o M-Learning. Por otro lado, no es suficiente contar con la infraestructura para reducir la brecha digital, se hace necesario la apropiación de las tecnologías por parte de los profesores para pensar en la aplicación en tiempos de pandemia de los nuevos enfoques como el STEAM, de tal manera que estudiantes y profesores interactúen virtualmente de manera sincrónica o asíncrona en espacios de aprendizajes con todas las garantías (HERRERÍA, 2020) (livari, Sharma, \& Ventä-Olkkonen, 2020).

Igualmente, desarrolladores e ingenieros han fortalecido una línea de trabajo de la ingeniería y la tecnología en torno a la educación, con el propósito de crear nuevas herramientas lúdicas para incorporar en el proceso de aprendizaje y enseñanza desde el enfoque STEAM. A su vez, la impresión 3D, las aplicaciones de drones, la analítica de datos y la inteligencia artificial, el uso de energías verdes y renovables son algunas de las tecnologías emergentes que los académicos e investigadores continúan estudiando con la finalidad de llevarlas a un nivel de madurez y confianza que masifique su utilización (Boza Muñoz, 2020) (Botero Silva, 2020).

La aparición del COVID -19 en un momento de transición entre la denominada Industria 3.0 y la Industria 4.0 ocasionó que se catapultaran algunos temas hasta ahora poco relevantes a nivel empresarial, y consigo generó un cambio de paradigmas que facilitaron la iniciación de la transformación digital en las organizaciones industriales y administrativas que aunque sea transitoria y motivada por la emergencia se prevé que serán adoptadas de manera permanente como una nueva normalidad, una vez se conozcan las bondades y beneficios que trae a las organizaciones. Para el caso del sector educativo, la transición a la industria 4.0 generó que los países desarrollados transformaran sus procesos educativos, especialmente a nivel curricular para integrar el enfoque STEAM (Pérez, 2020) (García-Peñalvo, Corell, Abella-García, \& Grande, 2020) (Oldekop et al., 2020) (Papadopoulos, Baltas, \& Balta, 2020).

En relación con lo descrito anteriormente, la transformación digital generada a partir del COVID-19 aportó a la disminución de la brecha digital en todos los sectores al generar más espacios para el teletrabajo, para la educación en línea y para la telemedicina, igualmente aportó a la consolidación de tecnologías basadas en inteligencia artificial, al crecimiento de la infraestructura médica de los países y al mejoramiento de plataformas digitales y aplicaciones móviles (García Peñalvo, 2020). Por tanto, al hacer un análisis prospectivo de las circunstancias actuales de la sociedad del conocimiento y la información, se puede proyectar que las tecnologías denominadas emergentes se consolidarán como tecnologías maduras en el corto plazo e iniciará una nueva era digital en el marco de una sociedad cibernética dependiente de los espacios ciber-físicos y conscientes de las bondades de la transformación Digital en las organizaciones.

Así mismo, las investigaciones y desarrollos científicos y tecnológicos en el marco de la pandemia han promovido un cambio de paradigma a nivel económico, social, cultural y de educación. Desde lo económico se espera un replanteamiento del modelo económico e industrial que permita prevenir a las organizaciones de escenarios de futuras pandemias (Braña Pino, 2020). También, es necesario plantear una etapa de adaptación tecnológica de acuerdo con el auge del mercado laboral y educativo desde el concepto de teletrabajo y educación en línea, por 
lo que es pertinente rediseñar el mundo actual, reorganizar el sistema y basado en el aprendizaje adquirido en estos tiempos difíciles, reiniciar y reconstruir, no obstante, esto se logrará si la educación es el pilar fundamental de esa nueva sociedad pos-pandemia, porque requerirá capacitarse y formarse en el uso de nuevas tecnologías . (Zafra Rodríguez, 2020) (Rivero \& Mota, 2020) (Iglesias Vidal, González-Patiño, Lalueza, \& Esteban-Guitart, 2020)

\section{Metodología}

El diseño metodológico utilizado fue de enfoque cualitativo, transeccional y de campo, Se entrevistaron a expertos, cuyo perfil profesional se orienta a ser profesores con más de 15 años de experiencia docente, adscritos a instituciones de educación en Colombia, con trayectoria en cada una de las áreas de la educación. Se utilizó la técnica de análisis de contenido, entrevista a profundidad y análisis de lenguaje natural mediante la minería de opiniones. La escala utilizada en la matriz de análisis fue una escala Likert con niveles cuantitativas de 0 a 5 y cualitativos Negativo, Ligeramente negativo, Neutro, Ligeramente positivo, Positivo. Esta escala se presenta en la tabla 1.

Tabla 1

Escala de conversión cualitativa - cuantitativa de postura de los expertos

\begin{tabular}{ccccc}
\hline \multicolumn{5}{c}{ Escala Likert utilizada } \\
\hline Negativo & $\begin{array}{c}\text { Ligeramente } \\
\text { Negativo }\end{array}$ & Neutral & $\begin{array}{c}\text { Ligeramente } \\
\text { Positivo }\end{array}$ & Positivo \\
\hline 0 & 1 & 2 & 3 & 4 \\
\hline
\end{tabular}

Para el análisis de contenido de la entrevista se utilizó una matriz de factores correlacionada con las respuestas de los expertos. El experto uno presentó un reconteo de palabras de 1529, una densidad léxica del 90\%, una diversidad léxica del $36 \%$ y una opinión o postura promedio Neutral. El experto dos presentó un reconteo de palabras de 576 , una densidad léxica del $85 \%$, una diversidad léxica del $41 \%$ y una opinión o postura promedio Neutral. El experto tres presentó un reconteo de palabras de 907, una densidad léxica del 90\%, una diversidad léxica de un $41 \%$, con una opinión o postura promedio Neutral. El experto cuatro presentó un reconteo de palabras de 1040, una densidad léxica del $89 \%$, una diversidad léxica del $39 \%$ y con una opinión promedio Neutral. Los datos presentados se encuentran en la tabla 2 y se identificaron con el fin de determinar la confiabilidad de la entrevista.

Tablam 2

Validación encuesta a profundidad

\begin{tabular}{l|ccccc}
\hline & $\begin{array}{c}\text { Recuento de } \\
\text { Palabras }\end{array}$ & Facilidad de lectura & Densidad léxica & Diversidad léxica & $\begin{array}{c}\text { Sentimiento de la } \\
\text { escritura }\end{array}$ \\
\hline Experto 1 & 1529 & $30 \%$ & $90 \%$ & $36 \%$ & Neutral \\
\hline Experto 2 & 576 & $30 \%$ & $85 \%$ & $41 \%$ & Neutral \\
\hline Experto 3 & 907 & $25 \%$ & $90 \%$ & $41 \%$ & Neutral \\
\hline Experto 4 & 1040 & $30 \%$ & $89 \%$ & $39 \%$ & Neutral \\
\hline
\end{tabular}

\section{Resultados}

De acuerdo con el estudio planteado sobre el covid-19 y su incidencia en la educación, se realizaron entrevistas a profundidad a cuatro expertos en educación. La entrevista abordó 10 aspectos relevantes de la educación en tiempos de pandemia con fines de relacionarlos con el estudio exploratorio sobre la educación en el contexto del enfoque STEAM, la industria 4.0 y la transformación digital. 
El primer factor analizado fue la incidencia del COVID-19 en el proceso de enseñanza, de este aspecto los expertos manifestaron que el impacto fue alto debido a que la enseñanza tiene una tradición de carácter presencial y a su vez no todos los docentes estaban familiarizados con la educación en línea y/o virtual, por lo cual, la no presencialidad impactó por el nulo contacto físico entre profesores y estudiantes, no obstante, hay una característica propuesta por una directiva docente enmarcada en la reinvención de los procesos y de las practicas pedagógicas.

De lo descrito anteriormente se puede analizar que los docentes de instituciones presenciales perciben que la pandemia generó un alto impacto en la enseñanza, que la virtualidad no permite profundizar en las temáticas de la misma manera como se hace en la presencialidad y que la falta de contacto humano presencial es fundamental para fortalecer los procesos educativos. Desde la mirada de la administración educativa se considera que aunque la medida transitoria a la virtualidad afectó en un grado alto, es posible hacer una reingeniería si se acude a metodologías nuevas y activas, acordes para un proceso de aprendizaje virtual o mixto que evite o minimice la fatiga de los estudiantes antes las arduas jornadas frente a la radiación que genera un equipo de cómputo y también estrategias que permitan trabajar las dimensiones del ser de los estudiantes frente a la digitalización de la educación.

El segundo factor analizado fue el relacionado con los cambios organizacionales que deben hacer las instituciones educativas ante el covid19, los expertos manifestaron que las instituciones educativas deben realizar cambios organizacionales desde lo académico y lo administrativo. Desde lo académico deben analizar el tiempo definido para las jornadas sincrónicas, ya que para docentes y estudiantes puede generar problemas de salud el tiempo de exposición frente al computador. Desde lo administrativo, la institución debe cambiar lo relacionado con la infraestructura tecnológica, la actualización de plataformas, la capacitación a los docentes, la dotación que facilite la implementación de una política de teletrabajo y la ampliación de las capacidades de la red de internet respecto al ancho de banda.

Con relación a lo manifestado por los expertos se rescata que las instituciones deben implementar un plan de transformación digital organizacional que permita el acondicionamiento digital para ejercer la función docencia y la función administrativa. Desde la docencia se debe hacer un proceso de concienciación a los estudiantes sobre la modalidad y lo que implica el autoaprendizaje, se deben capacitar a los docentes en el uso y apropiación de nuevas tecnologías que faciliten la generación de contenidos para aplicarlos en el aprendizaje asíncrono. Por su parte, desde lo administrativo las instituciones deben incorporar políticas, planes y estrategias de transformación digital que favorezcan la implementación de procesos y procedimientos más ágiles y eficientes; a su vez se debe avanzar en la apropiación de un modelo de teletrabajo que garantice el desarrollo de la modalidad mixta o blended learning en los procesos educativos. Todo lo anterior requiere de la inversión en infraestructura tecnológica para mejorar la conectividad, mejorar el acceso a tecnología de punta por parte de docentes y estudiantes.

El tercer factor de análisis fue la percepción de los expertos sobre la transición de la educación en línea por emergencia, desde la visión docencia los expertos convergen en que la educación en línea por emergencia fue buena e interesante y desde la visión de directivo docente manifiesta que se ha trabajado con normalidad. No obstante, las dos visiones comparten que existen obstáculos centrados en la adquisición de tecnologías por parte de docentes y estudiantes y obstáculos relacionados con una carga laboral muy alta.

De las percepciones de los expertos se evidencia que la educación en línea en tiempos de COVID-19 fue una alternativa de solución a un problema de contexto global, y aunque la educación en línea requiere unas habilidades diferentes a la educación presencial, la experiencia ha sido interesante y buena, esto concuerda con 
lo que académicos han encontrado en diferentes contextos frente a la educación Blended Learning, la cual combina las ventajas de la educación presencial con las de la educación en línea logrando una sinergia potente para el desarrollo de los procesos educativos. No obstante, también se evidencia que existen obstáculos relacionados con la escaza y costosa infraestructura tecnológica con la que cuentan las instituciones educativas y las familias de los estudiantes, lo que puede generar brechas sociales mayores a las actuales; sin embargo, esto también es una oportunidad para generar nuevas estrategias y tecnologías de bajo costo que apoyen al sector educativo y de esta manera hacer el tránsito a la educación dual. Por ello, de acuerdo con el estudio exploratorio se recomienda la incorporación del enfoque STEAM en los procesos educativos de las instituciones y el ajuste de los currículos al contexto de la industria 4.0.

El cuarto factor analizó la vivencia de estudiantes y profesores en medio de la educación en línea o remota por emergencia ante la pandemia, los expertos manifestaron que tanto estudiantes como docentes han asumido la transición de la metodología con un alto compromiso, que, aunque inicialmente los estudiantes percibían el cambio solo como algo novedoso, con el paso del tiempo la gran mayoría ha mostrado empatía por las clases mediadas por tecnologías. Sin embargo, se menciona lo complejo de la situación debido a que se necesita más preparación de contenido, mejor infraestructura tecnológica, mayor capacidad de ancho de banda en la conectividad, entre otros. También se evidencia que en algunos casos los estudiantes no cuentan con la tecnología necesaria para afrontar la educación desde la virtualidad.

De acuerdo con lo manifestado por los expertos y al contrastarlo con la literatura especializada se evidencia que tanto docentes y estudiantes han afrontado el nuevo escenario de la educación en línea y remota con entusiasmo y gran compromiso, sin embargo en algunos casos la motivación disminuye con el pasar del tiempo y para otros casos la motivación continua y genera empatía por el aprendizaje mediado por tecnologías, también se evidencia que los docentes están obligados a trabajar mas tiempo debido a la necesidad de crear contenidos digitales como apoyo a la docencia, así mismo, se vuelve a mencionar el déficit tecnológico en infraestructura y conectividad. Por ello, se recomienda generar nuevas estrategias didácticas acompañadas de nuevas metodologías para apoyar el proceso de aprendizaje mediado por las TIC y se reitera la oportunidad para aplicar el enfoque STEAM como método para solucionar problemas del contexto mediante la integración de las ciencias, la tecnología, la ingeniería, las artes y las matemáticas.

Un quinto factor de análisis fue sobre la pertinencia de la educación Blended Learning en los niveles de primera infancia, primaria y secundaria. Los expertos manifestaron que en el nivel de educación básica secundaria y media hay una alta probabilidad de implementación, mientras que consideran que en el nivel de primaria y primera infancia es menos pertinente por las edades de los estudiantes, debido a que la virtualidad no garantiza un pleno desarrollo de la personalidad en los niños y ellos requieren el contacto con sus pares de tal forma que ayude al proceso formativo del ser.

En concordancia con los expertos y la literatura científica, existe una preocupación por la implementación de la educación mixta combinada o Blended learning en los niveles educativos de primera infancia y primaria, puesto que la integración y el contacto físico con profesores y estudiantes es necesario para la construcción de la personalidad y del carácter de los niños. No obstante, otros investigadores manifiestan que, si se capacita a los padres de familia, a los profesores, y se brindan entornos virtuales de aprendizaje adaptativos digitalmente, es posible una implementación de la metodología combinada. También, se evidencia que ante una sociedad que estará cada día mas expuesta a enfermedades del ambiente como la mala calidad del aire, es necesario prepararse para una cultura diferente, una cultura de una sociedad tecnológica, con nuevos hábitos y diversas personalidades que posiblemente aun no se conocen. 
El sexto factor de análisis fue sobre las nuevas estrategias curriculares y educativas que se deben implementar en el campo educativo para afrontar la pospandemia, los expertos manifestaron que se requieren estrategias que permitan a las organizaciones educativas contar con mayor solvencia presupuestal y minimizar la contratación con terceros y que estén encaminadas al mejoramiento de la infraestructura tanto física como digital. Igualmente, se necesitan estrategias de flexibilización del currículo, de cualificación docente permanente, y estrategias psicopedagógicas que permitan a los niños afrontar la nueva realidad tras la pandemia.

En el análisis realizado se evidencia que el sector educativo debe trabajar en el desarrollo de nuevas estrategias que impactan el ámbito curricular, pedagógico y administrativo de tal forma que la comunidad académica retome la confianza ante las profundas transformaciones sociales que ocasionó la pandemia.

En el factor siete denominado $\boldsymbol{A B P}$ y transformación digital en la educación, los expertos manifestaron que el aprendizaje basado en problemas o proyectos. fortalece el proceso de enseñanza - aprendizaje en el aula de clase, pero a su vez requiere fortalezas en las competencias en tecnología. Adicionalmente, la integración de las TICs y la transformación digital dentro de los procesos académico-administrativos y las áreas curriculares sería una estrategia importante para preparar a los estudiantes y al personal docente en caso de que se presente una situación similar a la que se vive actualmente. También manifestaron la importancia de que las instituciones educativas experimenten un proceso de transformación digital en las diferentes funciones misionales de la misma.

En este factor se evidencia que el aprendizaje basado en problemas y proyectos es pertinente en la educación presencial y virtual ya que facilita el desarrollo del pensamiento espacial, crítico y computacional, ayuda a la toma de decisiones asertivas y aporta a la integración de las demás áreas. Así mismo, se evidencia que es ineludible un plan de transformación digital que permita la incorporación de tecnologías digitales a la administración educativa, al currículo y a las estrategias didácticas. Por lo anterior, se recomienda que se trabaje en un proceso de innovación educativa en todos los niveles de tal forma que se incorporen los elementos mencionados anteriormente, para esto es necesario cambiar el currículo centrados en ciencias a un currículo STEAM que permita el desarrollo de habilidades para el siglo XXI y necesarias para el aprendizaje a lo largo de la vida como lo plantea UNESCO.

Por otra parte, la investigación planteó un análisis subjetivo referente a la opinión de los expertos con el fin de conocer sus estados emocionales frente a un tema tan susceptible como el de pandemia y poder encontrar resultados que indicaran desde el procesamiento del lenguaje natural la postura negativa, neutra o positiva frente el tema objeto de estudio. La tabla 1, presenta la escala de conversión cuantitativa - cualitativa utilizada.

El factor uno presenta un nivel de 1.8 equivalente a una postura neutra con tendencia hacia lo negativo referente a la incidencia del COVID -19 en los procesos de enseñanza y aprendizaje, para el factor dos, el análisis cualitativo evidencia que la postura promedio de los expertos frente a los cambios organizacionales de las instituciones educativas frente al COVID -19 es de un nivel de 2.0 equivalente a una postura neutral, el factor tres, presenta un nivel de 1.5 lo que indica que la tendencia se encuentra entre lo ligeramente negativo y neutral ante la educación en línea como alternativa ante la emergencia sanitaria, así mismo, el factor cuatro, entregó un nivel de 2.3 lo que indica que frente a como han afrontado los estudiantes y profesores la educación online por emergencia la postura de los expertos se encuentra entre neutral y ligeramente positiva. Para el factor cinco, el nivel arrojó un valor de 1.0 lo que indica que los expertos tienen una postura ligeramente negativa frente a la implementación de una educación mixta o combinada (virtual y presencial) después de la pandemia. El factor 6 , presenta una postura ligeramente negativa frente a las estrategias curriculares y educativas para implementar en el campo educativo al finalizar la pandemia. Finalmente, el factor siete obtuvo un valor de 2.0 lo que indica que la postura es neutral frente al desarrollo del ABP y transformación digital en la educación. La tabla 3 y 4 , presentan los resultados descritos anteriormente. 
Tabla 3

Resultados cuantitativos de lenguaje natural mediante minería de opinión

\begin{tabular}{cccccccc}
\hline & \multicolumn{7}{c}{ Factor de análisis } \\
\hline Experto 1 & 1 & 2 & 3 & 4 & 5 & 6 & 7 \\
Experto 2 & 0,0 & 2,0 & 2,0 & 2,0 & 0,0 & 0,0 & 2,0 \\
Experto 3 & 3,0 & 0,0 & 4,0 & 3,0 & 2,0 & 0,0 & 2,0 \\
Experto 4 & 2,0 & 2,0 & 0,0 & 4,0 & 1,0 & 2,0 & 2,0 \\
Media & 1,8 & 4,0 & 0,0 & 0,0 & 1,0 & 2,0 & 2,0 \\
\hline
\end{tabular}

Tabla 4

Resultados cualitativos de lenguaje natural mediante minería de opinión

\begin{tabular}{|c|c|c|c|c|c|c|c|}
\hline & \multicolumn{7}{|c|}{ Factor de análisis } \\
\hline & 1 & 2 & 3 & 4 & 5 & 6 & 7 \\
\hline Experto 1 & $\begin{array}{l}\text { Sentimiento } \\
\text { Negativo }\end{array}$ & $\begin{array}{c}\text { Sentimiento } \\
\text { Neutral }\end{array}$ & $\begin{array}{c}\text { Sentimiento } \\
\text { Neutral }\end{array}$ & $\begin{array}{c}\text { Sentimiento } \\
\text { Neutral }\end{array}$ & $\begin{array}{l}\text { Sentimiento } \\
\text { Negativo }\end{array}$ & $\begin{array}{l}\text { Sentimiento } \\
\text { Negativo }\end{array}$ & $\begin{array}{c}\text { Sentimiento } \\
\text { Neutral }\end{array}$ \\
\hline Experto 2 & $\begin{array}{l}\text { Sentimiento } \\
\text { Ligeramente } \\
\text { Positivo }\end{array}$ & $\begin{array}{l}\text { Sentimiento } \\
\text { Negativo }\end{array}$ & $\begin{array}{c}\text { Sentimiento } \\
\text { Positivo }\end{array}$ & $\begin{array}{l}\text { Sentimiento } \\
\text { Ligeramente } \\
\text { Positivo }\end{array}$ & $\begin{array}{c}\text { Sentimiento } \\
\text { Neutral }\end{array}$ & $\begin{array}{c}\text { Sentimiento } \\
\text { Negativo }\end{array}$ & $\begin{array}{c}\text { Sentimiento } \\
\text { Neutral }\end{array}$ \\
\hline Experto 3 & $\begin{array}{l}\text { Sentimiento } \\
\text { Neutral }\end{array}$ & $\begin{array}{l}\text { Sentimiento } \\
\text { Neutral }\end{array}$ & $\begin{array}{l}\text { Sentimiento } \\
\text { Negativo }\end{array}$ & $\begin{array}{l}\text { Sentimiento } \\
\text { Positivo }\end{array}$ & $\begin{array}{l}\text { Sentimiento } \\
\text { Ligeramente } \\
\text { Negativo }\end{array}$ & $\begin{array}{c}\text { Sentimiento } \\
\text { Neutral }\end{array}$ & $\begin{array}{c}\text { Sentimiento } \\
\text { Neutral }\end{array}$ \\
\hline Experto 4 & $\begin{array}{l}\text { Sentimiento } \\
\text { Neutral }\end{array}$ & $\begin{array}{l}\text { Sentimiento } \\
\text { Positivo }\end{array}$ & $\begin{array}{l}\text { Sentimiento } \\
\text { Negativo }\end{array}$ & $\begin{array}{l}\text { Sentimiento } \\
\text { Negativo }\end{array}$ & $\begin{array}{c}\text { Sentimiento } \\
\text { Ligeramente } \\
\text { Negativo }\end{array}$ & $\begin{array}{c}\text { Sentimiento } \\
\text { Neutral }\end{array}$ & $\begin{array}{c}\text { Sentimiento } \\
\text { Neutral }\end{array}$ \\
\hline Media & $\begin{array}{l}\text { Sentimiento } \\
\text { Negativo }\end{array}$ & $\begin{array}{c}\text { Sentimiento } \\
\text { Neutral }\end{array}$ & $\begin{array}{c}\text { Sentimiento } \\
\text { Neutral }\end{array}$ & $\begin{array}{l}\text { Sentimiento } \\
\text { Neutral }\end{array}$ & $\begin{array}{c}\text { Sentimiento } \\
\text { Negativo }\end{array}$ & $\begin{array}{c}\text { Sentimiento } \\
\text { Negativo }\end{array}$ & $\begin{array}{c}\text { Sentimiento } \\
\text { Neutral }\end{array}$ \\
\hline
\end{tabular}

\section{Conclusiones}

La implementación de un plan estratégico de transformación digital en las organizaciones educativas permitirá mejorar significativamente los procesos de enseñanza y aprendizaje en los diferentes niveles de formación, Así como preparar a las futuras generaciones para el contexto de la industria 4.0 en el marco de la era digital.

La adecuación del currículo STEAM en las instituciones educativas de educación básica y media, permitirá articular la formación de la educación media y universitaria, con fines de desarrollar las habilidades necesarias para el siglo XXI, especialmente en habilidades para la toma de decisiones, pensamiento crítico, pensamiento computacional y estar preparado con una visión holística a lo largo de la vida.

En la década de la era digital las organizaciones deben adaptar políticas e infraestructura que permitan mejoras continuas de los procesos educativos. Para esto es necesario innovar en plataformas educativas, ecosistemas de negocios, productos de múltiple finalidad, cooperación estratégica entre otros.

El contexto de la industria 4.0 requiere procesos educativos disruptivos por tanto es necesario aplicar metodologías activas que permitan mejorar los procesos didácticos de la formación, a su vez, hacer uso y apropiación de tecnologías emergentes que permitan el desarrollo de contenidos digitales pertinentes para las exigencias de la cuarta revolución industrial. 
La educación presente y futura de la sociedad afectada por el COVID-19 requiere un proceso de reingeniería de tal forma que involucre políticas, planes y estrategias de transformación digital, que permita apropiar nuevos enfoques educativos como el STEAM y nuevas metodologías activas apropiadas para mediaciones en las diferentes formas del aprendizaje mediado por tecnologías digitales.

Se evidencio que para afrontar una educación en tiempos de Covid-19, es necesario incorporar estrategias curriculares y educativas nuevas de acuerdo a las necesidades de contexto. No obstante, una de las estrategias mas pertinentes para la educación en tiempos de pandemia es el ABP apropiando proceso de transformación digital en las organizaciones educativas.

\section{Referencias bibliográficas}

Argudo, M. del C. C., \& Tenecela, M. C. P. (2019). Educación con Tecnología en una Pandemia: Breve Análisis. Journal of Chemical Information and Modeling, 53(9), 1689-1699.

Boza Muñoz, S. (2020). Diseño y prototipado de un dron aéreo desmontable para niños mediante tecnologías DIY (Universitat Politècnica de València. Facultad de Bellas Artes). Recuperado de http://hdl.handle.net/10251/148491

Botero Silva, D. carolina. (2020). Automatización y género ¿Es la automatización laboral un factor que profundiza o reduce la inequidad de género? El caso de la industria automotriz en México a 2035. Escuela de gobierno y transformacion publica. https://doi.org/https://hdl.handle.net/11285/636550

García-Peñalvo, F. J., Corell, A., Abella-García, V., \& Grande, M. (2020). Online assessment in higher education in the time of COVID-19. Education in the Knowledge Society, 21, 1-26.

https://doi.org/10.14201/eks.23013

Habib, H., González, C., Collazos, C. A., \& Yousef, M. (2020). Estudio exploratorio en iberoamérica sobre procesos de enseñanza-aprendizaje y propuesta de evaluación en tiempos de pandemia. Education in the Knowledge Society (EKS), 21(0), 9. https://doi.org/10.14201/eks.23537

Dayekh Garcia, A. (2020). Proyecto steam en base a videojuegos comerciales un despliegue interdisplinar de las matemáticas, la ciencia y el arte "arcade steam". Universidad de la Laguna.

Iglesias Vidal, E., González-Patiño, J., Lalueza, J. L., \& Esteban-Guitart, M. (2020). Manifiesto en Tiempos de Pandemia: Por una Educación Crítica, Intergeneracional, Sostenible y Comunitaria. Revista Internacional de Educación para la Justicia Social, 9(3), 181-198. https://doi.org/10.15366/riejs2020.9.3.010

livari, N., Sharma, S., \& Ventä-Olkkonen, L. (2020). Digital transformation of everyday life - How COVID-19 pandemic transformed the basic education of the young generation and why information management research should care? International Journal of Information Management, (June), 102183. https://doi.org/10.1016/j.ijinfomgt.2020.102183

Oldekop, J. A., Horner, R., Hulme, D., Adhikari, R., Agarwal, B., Alford, M., ... Zhang, Y. F. (2020). COVID-19 and the case for global development. World Development, 134, 105044. https://doi.org/10.1016/j.worlddev.2020.105044

Papadopoulos, T., Baltas, K. N., \& Balta, M. E. (2020). The use of digital technologies by small and medium enterprises during COVID-19: Implications for theory and practice. International Journal of Information Management, (June), 102192. https://doi.org/10.1016/j.ijinfomgt.2020.102192

Pérez, E. L. (2020). La industria 4.0 y las nuevas formas de trabajar: una perspectiva desde el caso mexicano en tiempos de covid 19. Lan Harremanak - Revista de Relaciones Laborales, 43, 17. 
Rivero, P. A. C., \& Mota, M. del P. E. (2020). Evolución de las habilidades laborales en la industria 4.0 y su impacto financiero. Revista Innova ITFIP, 6(1), 106-119

Wosik, J., Fudim, M., Cameron, B., Gellad, Z. F., Cho, A., Phinney, D., ... Tcheng, J. (2020). Telehealth transformation: COVID-19 and the rise of virtual care. Jedrek Wosik, Marat Fudim, Blake Cameron, Ziad F. Gellad, Alex Cho, Donna Phinney, Simon Curtis, Matthew Roman, Eric G. Poon, Jeffrey Ferranti, Jason N. Katz, James Tcheng, 27(6), 957-962. https://doi.org/10.1093/jamia/ocaa067

Zafra Rodríguez, C. A. (2020). Aprendizaje de la automatización industrial en tiempos de pandemia. Una Experiencia virtual de aprendizaje basado en proyectos. (Universidad Agustiniana Facultad de Humanidades, Ciencias Sociales y Educación).

Esta obra está bajo una Licencia Creative Commons Attribución-NoCommercial 4.0 International

\section{(cc) EY-NC}

